\title{
Shear Viscosity at Infinite Coupling: A Field Theory Calculation
}

\author{
Paul Romatschke \\ Department of Physics, University of Colorado, Boulder, Colorado 80309, USA \\ and Center for Theory of Quantum Matter, University of Colorado, Boulder, Colorado 80309, USA
}

(Received 22 April 2021; accepted 13 August 2021; published 9 September 2021)

\begin{abstract}
I derive an exact integral expression for the ratio of shear viscosity over entropy density $\eta / s$ for the massless (critical) $O(N)$ model at large $N$ with quartic interactions. The calculation is set up and performed entirely from the field theory side using a nonperturbative resummation scheme that captures all contributions to leading order in large $N$. In $2+1 d, \eta / s$ is evaluated numerically at all values of the coupling. For infinite coupling, I find $(\eta / s) \simeq 0.42(1) \times N$. I show that this strong coupling result for the viscosity is universal for a large class of interacting bosonic $O(N)$ models.
\end{abstract}

DOI: 10.1103/PhysRevLett.127.111603

The shear viscosity is a transport coefficient that encodes how efficiently spatial anisotropies are transmuted into momentum anisotropies (velocity gradients). For relativistic systems, a key dimensionless ratio that quantifies this efficiency is found by the ratio of shear viscosity $\eta$ to entropy density $s$. Experimental measurements of momentum anisotropies in heavy-ion collisions together with hydrodynamic modeling constrain the value of shear viscosity in QCD to $(\eta / s) \lesssim 0.2[1-5]$.

This numerical value happens to be not too dissimilar from the result $(\eta / s)=(1 / 4 \pi) \simeq 0.08$ found for the conjectured strong-coupling limit of another gauge theory, $\mathcal{N}=4$ supersymmetric Yang-Mills theory, in the large $N$ limit [6-8]. By contrast, in QCD where calculations are limited to weak coupling only, one typically encounters values $(\eta / s) \gg(1 / 4 \pi)[9-11]$.

At intermediate couplings, results for the shear viscosity exist for QED with many fermion flavors [12] and for SU(3) gauge theory from lattice Monte Carlo simulations [13,14]. At strong coupling, results for transport coefficients have been limited to theories with known holographic duals, with the exception of so-called thermodynamic transport coefficients, such as $\kappa$ [15]. In particular, there is no known example of a theory where $\eta / s$ has been determined for all coupling strengths.

The present Letter is meant to fill this gap and provide the complete coupling dependence for the shear viscosity over entropy ratio by directly calculating the relevant lowfrequency limit of the energy-momentum tensor correlator from the field theory. The theory to be studied will be the

Published by the American Physical Society under the terms of the Creative Commons Attribution 4.0 International license. Further distribution of this work must maintain attribution to the author(s) and the published article's title, journal citation, and DOI. Funded by SCOAP.
$O(N)$ vector model with quartic interactions in $2+1$ dimensions in the large $N$ limit, which exists for all values of the coupling. The choice of this theory is motivated by the fact that the entropy density $s$ is known for all couplings [16] and that an efficient resummation scheme that captures all relevant contributions to the shear viscosity at large $N$ is known $[17,18]$. It also helps that the most difficult part of the calculation, namely, the evaluation of the shear viscosity coefficient for the $O(N)$ vector model, has already been set up for the $3+1 d$ theory using a variant of the twoparticle irreducible formalism in Ref. [19]. Thus, strictly speaking, the only new result in the present Letter will be the determination of $\eta / s$ at infinite coupling, which can be done in the $3 d$ large $N O(N)$ model (but does not make sense because of the Landau pole in $3+1 d$ ).

A major objection to calculating the shear viscosity in a theory with only two space dimensions is the presence of so-called long-time tails [20], which normally lead to a divergent two-dimensional shear viscosity when naively taking the low-frequency limit. However, for the $O(N)$ model it so happens that $(\eta / s) \propto N$, so that in the large $N$ limit long-time tails are suppressed by three powers of $N$, cf. the discussion in Ref. [21]. For this reason, the calculation of the shear viscosity as the zero-frequency limit of the relevant energy-momentum correlator reported in this Letter is well defined.

Preliminaries.-In the interest of brevity, I will not review the setup of finite-temperature correlators in quantum field theory (the interested reader is referred to the Supplemental Material for this topic [22]). Denoting Minkowski momenta as $\mathcal{K}=(\omega, \mathbf{k})$ and space-time coordinates as $\mathcal{X}=(t, \mathbf{x})$, I will use the relation of retarded real-time Green's functions $G_{R}$ and the spectral density $\rho$ given by

$$
G_{R}(\mathcal{K})=-\int \frac{d \mu}{\pi} \frac{\rho(\mu, \mathbf{k})}{\mu-\omega-i 0^{+}}
$$


This relation can be used to derive the analytic continuation of the corresponding Euclidean correlator to real time (cf. Refs. [23,24])

$$
G_{R}(\omega)=-G_{E}\left(\omega_{E} \rightarrow i \omega-0^{+}\right) .
$$

In order to connect the retarded correlator with transport coefficients, one employs the low-momentum expansion of $G_{R}$ provided by fluid dynamics. Fluid dynamics is the effective field theory (EFT) of conserved quantities for small moment. For a theory that conserves energy and momentum, good EFT variables are the energy density and fluid four-velocity $\epsilon, u^{\mu}$, fulfilling $u^{\mu} u_{\mu}=-1$ (see, e.g., Ref. [23-25] for reviews of modern fluid dynamics). Using fluid dynamics, it is straightforward to derive the relation $G_{R}(\omega, \mathbf{k}=0)=P-i \omega \eta+\mathcal{O}\left(\omega^{2}\right)$ for the $T^{x y}$ correlator in $d \geq 3$ space-time dimensions, from which the so-called Kubo relation follows,

$$
\lim _{\omega \rightarrow 0} \frac{\partial}{\partial \omega} \rho^{x y, x y}(\omega, \mathbf{k}=0)=\eta .
$$

Including thermal fluctuations in the fluid dynamic calculations leads to a long-time tail contribution for $d=3$ of the form $G_{R}(\omega, \mathbf{k}=0) \propto\left[i \omega T^{2} /(\eta / s)\right] \ln \omega$ (see Supplemental Material [22]). This term, in general, invalidates the Kubo formula (3), except in the large $N$ limit where it is suppressed by $(\eta / s) \propto N$, thereby allowing the use of (3) to extract the shear viscosity for $d=3$ to $\mathcal{O}\left(N^{0}\right)$.

The $O(N)$ model.- The field theory I consider in this Letter is that of a massless ("critical") $N$-component scalar field $\phi_{a}, a=1,2, \ldots, N$ with Euclidean action

$$
S=\int d^{d} X\left[\frac{1}{2} \partial_{\mu} \phi_{a} \partial_{\mu} \phi_{a}+\frac{\lambda}{N}\left(\phi_{a} \phi_{a}\right)^{2}\right],
$$

where at finite temperature $T$ the $X^{0}=\tau$ direction is compactified on a circle with radius $\beta$. The partition function for this theory $Z=\int \mathcal{D} \phi e^{-S}$ may be rewritten by inserting $1=\int \mathcal{D} \sigma \delta\left(\sigma-\phi_{a} \phi_{a}\right)=\int \mathcal{D} \sigma \mathcal{D} \zeta e^{i \int \zeta\left(\sigma-\phi_{a} \phi_{a}\right)}$. Integrating out the $\sigma$ field gives

$$
Z=\int \mathcal{D} \phi \mathcal{D} \zeta e^{-\int_{X}\left(\frac{1}{2} \partial_{\mu} \phi_{a} \partial_{\mu} \phi_{a}+i \zeta \phi_{a} \phi_{a}+\frac{N}{4 h} \zeta^{2}\right)}
$$

Splitting the field $\zeta=\frac{1}{2} \zeta_{0}+\zeta^{\prime}$ into a zero mode and fluctuations, the action in (5) becomes

$$
\begin{aligned}
S & =S_{R 0,0}+S_{R 0, I}+\frac{N V \beta}{16 \lambda} \zeta_{0}^{2}, \\
S_{R 0,0} & =\frac{1}{2} \int_{X}\left(\partial_{\mu} \phi_{a} \partial_{\mu} \phi_{a}+i \zeta_{0} \phi_{a} \phi_{a}+\frac{N}{2 \lambda} \zeta^{\prime 2}\right),
\end{aligned}
$$

where $S_{R 0, I}=i \int_{X} \zeta^{\prime} \phi_{a} \phi_{a}$.
At leading order in large $N$, only the zero mode from the $\zeta$ field contributes to the partition function, so $S_{R 0, I}$ may be ignored. This corresponds to a particular resummation of finite-temperature Feynman diagrams (certain tadpoles), dubbed the $R 0$-level resummation in Ref. [17]. One finds for $R 0$,

$$
Z_{R 0}=\sqrt{\frac{N V \beta}{16 \pi \lambda}} \int d \zeta_{0} e^{-\frac{N V \beta}{16 \zeta^{2}} \zeta_{0}^{2}-N V \beta J\left(\sqrt{i \zeta_{0}}\right)}
$$

where $V$ is the $d$-1-dimensional volume of space and $e^{-N V \beta J\left(\sqrt{i \zeta_{0}}\right)} \equiv \int \mathcal{D} \phi e^{-\frac{1}{2} \int_{X}\left(\partial_{\mu} \phi_{a} \partial_{\mu} \phi_{a}+i \zeta_{0} \phi_{a} \phi_{a}\right)}$. In the large $N$ limit, the remaining ordinary integral in (7) can be evaluated exactly from the saddle point located at $i \zeta_{0}=m^{2}$, so that $Z_{R 0}=e^{-N V \beta\left[m^{4}-J(m)\right]}$, where

$$
J(m)=\frac{1}{2} \int \frac{d^{d-1} \mathbf{k}}{(2 \pi)^{d}}\left[E_{k}+2 T \ln \left(1-e^{-E_{k} \beta}\right)\right],
$$

$E_{k}=\sqrt{\mathbf{k}^{2}+m^{2}}$ ([26], Eq. (2.44)]. Using dimensional regularization for $d=3-0^{+}$, the saddle point condition becomes

$$
\pi \hat{\lambda} \hat{m}^{2}+\hat{m}+2 \ln \left(1-e^{-\hat{m}}\right)=0,
$$

where $\hat{m} \equiv \beta m, \hat{\lambda} \equiv \beta \lambda$. Note that in the strong-coupling limit $\hat{\lambda} \rightarrow \infty$ (9) has a universal solution $\hat{m}=2 \ln [(1+$ $\sqrt{5}) / 2][16,27]$. Basic thermodynamic relations give the exact large $N$ entropy density as $s=(\partial / \partial T)\left(\ln Z_{R 0} / \beta V\right)$. Using (9), integration by parts, and a variable change to $E=E_{k}$, for $d=3$ this relation leads to

$$
\begin{aligned}
s & =-\frac{N \beta}{4 \pi} \int_{m}^{\infty} d E E\left(E^{2}-m^{2}\right) n^{\prime}(E), \\
& =\frac{N T^{2}}{2 \pi}\left[2 \hat{m}^{2} \ln n(m)+6 \hat{m} \operatorname{Li}_{2}\left(e^{-\hat{m}}\right)+6 \operatorname{Li}_{3}\left(e^{-\hat{m}}\right)\right],
\end{aligned}
$$

where $n(x)=\left(e^{\beta x}-1\right)^{-1}$ and $n^{\prime}(x)=\partial_{x} n(x)$.

For some correlation functions, additional interactions not included in the $R 0$ resummation may contribute at leading order in large $N$, making it necessary to go beyond the $R 0$ level. To this end, slightly changing notation from Ref. [17], rewrite the action (6) as $S_{R 0,0}+S_{R 0, I}=S_{R 2,0}+$ $S_{R 2, I}$ with

$$
\begin{aligned}
& S_{R 2,0}=\frac{1}{2} \int_{X, Y}\left(\phi_{a} \Delta^{-1} \phi_{a}+\zeta^{\prime} D^{-1} \zeta^{\prime}\right), \\
& S_{R 2, I}=i \int_{X} \zeta^{\prime} \phi_{a} \phi_{a}-\frac{1}{2} \int_{X, Y}\left(\phi_{a} \Sigma \phi_{a}+\zeta^{\prime} N \Pi \zeta^{\prime}\right),
\end{aligned}
$$

where 
$\Delta(K)=\frac{1}{K^{2}+m^{2}+\Sigma(K)}, \quad D(K)=\frac{1}{N} \frac{1}{\frac{1}{2 \lambda}+\Pi(K)}$.

The $R 2$-level resummation then consists of calculating $\Sigma, \Pi$ self-consistently up to (including) one-loop level using $S_{R 2, I}$, finding

$$
\Sigma_{R 2}(X)=2 D(X) \Delta(X), \quad \Pi_{R 2}(X)=4 \Delta^{2}(X) .
$$

At large $N, D \propto(1 / N)$, so $\Sigma$ is generically subleading, while $\Pi$ is not.

At finite temperature, more subtle ways generating contributions at leading order in large $N$ arise, making it necessary to go beyond the $R 2$ resummation. To this end, rewrite the action (6) again using $S_{R 2,0}, S_{R 2, I}$ but express

$$
i \int_{X} \zeta^{\prime} \phi_{a} \phi_{a}=+i \int_{X, Y, Z} \zeta^{\prime} \phi_{a} \phi_{a}\left[\Gamma_{3}-\delta \Gamma_{3}\right]
$$

where $\Gamma_{3}(P, K)=1+\delta \Gamma_{3}(P, K)$ is the resummed threevertex function and $P$ and $K$ are the incoming momenta for the $\Delta$ propagators. The $R 3$-level resummation then consists of calculating $\Gamma_{3}$ self-consistently to one-loop level, and $\Sigma$, $\Pi$ self-consistently up to (including) two-loop level. Diagrammatically, one finds [17]

$$
\delta \Gamma_{3}=-4 \cdots \xi
$$

where the full vertex is denoted by

$$
\Gamma_{3}=m
$$

where wiggly lines denote $D(K)$ and straight lines denote $\Delta(K)$.

While the $R 0$ resummation contains all leading order large $N$ contributions for the zero-point function, $R 2$ and $R 3$ contain all large $N$ contributions for the two- and threepoint function, respectively. The energy-momentum tensor is a four-point function, so including all large $N$ contributions requires going to $R 4$. The $R 4$ resummation is found by adding and subtracting a term $\int_{X, Y, U, Z} \phi_{a} \phi_{a} \phi_{b} \phi_{b} \Gamma_{4}$. Calculating the one-loop four-point vertex self-consistently, one finds diagrammatically

$$
\Gamma_{4}=-2
$$

whereas the large $N$ three-point vertex in $R 4$ becomes

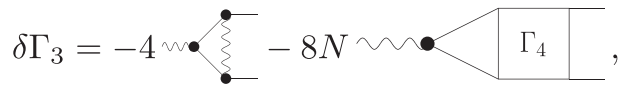

and the self-energies $\Pi, \Sigma$ are still diagrammatically given by (15). Note that since $D \propto N^{-1}$, the four-point vertex and the triangle diagram contribute at the same order at large $N$.

Energy-momentum tensor correlators. - For the action (4), the Euclidean operator for the energy-momentum tensor is given by $T^{x y}(X)=\partial_{x} \phi_{a}(X) \partial_{y} \phi_{a}(X)$ so that the Euclidean energy-momentum tensor correlator is defined by

$$
G_{E}^{x y, x y}(X)=\frac{\int \mathcal{D} \phi e^{-S} T^{x y}(X) T^{x y}(0)}{Z} .
$$

In the $R 0$-level resummation, following (6) and neglecting $S_{R 0, I}$, the $R 0$ action is quadratic in $\phi$ and hence $G_{E}^{x y, x y}$ can be calculated using Wick's theorem. At finite temperature, $\phi_{a}(X)=T \sum_{k_{E}} \int_{\mathbf{k}} e^{i K X} \phi_{a}(K)$, where $k_{E}=2 \pi n T$ are the bosonic Matsubara frequencies with $n \in \mathbb{Z}$. In Fourier space with $\mathbf{p}=p \hat{x}$, one finds $[15,16]$

$G_{E, R 0}^{x y, x y}(P)=2 N \bigcup_{K} \frac{\left(k_{x}-p\right)^{2} k_{y}^{2}}{\left(K^{2}+m^{2}\right)\left[(K-P)^{2}+m^{2}\right]}$,

where the mass $m$ is determined by (9). The result may be analytically continued to real frequencies $\omega$ using Eq. (2). The $R 0$-level result $G_{R, R 0}^{x y, x y}(\omega, \mathbf{p}=0)$ constitutes the correct large $N$ result for the retarded correlator except for the region $\beta \omega \ll(1 / N)$.

To appreciate this statement, let us reconsider $G_{E}^{x y, x y}$ in the $R 2$-level resummation. Using (11) and neglecting $S_{R 2, I}$, the $R 2$ action is once again quadratic in the fields, and hence $G_{E}^{x y, x y}$ can be calculated using Wick's theorem. Setting again $\mathbf{p} \rightarrow 0$ gives

$$
G_{E, R 2}^{x y, x y}\left(p_{E}\right)=2 N \bigcup_{K} k_{x}^{2} k_{y}^{2} \Delta(K) \Delta(P-K),
$$

with $\Sigma=\Sigma_{R 2}$ in (12) and $\Sigma_{R 2}$ specified by (13). Using the spectral representation of the propagator

$$
\rho(\mathcal{K})=-\frac{\operatorname{Im} \Sigma(\mathcal{K})}{\left[\mathcal{K}^{2}+m^{2}+\operatorname{Re} \Sigma(\mathcal{K})\right)^{2}+(\operatorname{Im} \Sigma(\mathcal{K})]^{2}},
$$

the thermal sum and angular integral is straightforward, giving for $d=3$

$$
\begin{aligned}
G_{E, R 2}^{x y, x y}\left(p_{E}\right)= & \frac{N}{4} \int_{0}^{\infty} \frac{d k k^{5}}{2 \pi} \int \frac{d \mu d \mu^{\prime}}{2 \pi^{2}} \frac{\rho(\mu) \rho\left(\mu^{\prime}\right)}{\mu+\mu^{\prime}+i p_{E}} \\
& \times\left(\operatorname{coth} \frac{\mu}{2 T}+\operatorname{coth} \frac{\mu^{\prime}}{2 T}\right) .
\end{aligned}
$$

Note that since $\Sigma \propto(1 / N)$, in the naive large $N$ limit (21) becomes the spectral function for a free massive particle, 
$\rho(\omega)=\pi \operatorname{sign}(\omega) \delta\left(\mathcal{K}^{2}+m^{2}\right)$. Continuing $G_{E}^{x y, x y}$ analytically to real frequencies $\omega_{E} \rightarrow i \omega-0^{+}$, the imaginary part becomes

$\rho_{R 2}^{x y, x y}(\omega)=\frac{N}{2} \int_{\mathcal{K}} \mathbf{k}^{4} \rho\left(k^{0}\right) \rho\left(\omega-k^{0}\right)\left[n\left(k^{0}\right)-n\left(k^{0}-\omega\right)\right]$.

One finds that the product $\rho\left(k^{0}\right) \rho\left(k^{0}-\omega\right)$ has contributions where poles "pinch" the real $k^{0}$ axis from above and below in the large $N$ limit. Focusing on the limit $\beta \omega \ll(1 / N)$, this contribution becomes

$$
\rho^{2}\left(k^{0}\right) \rightarrow \frac{1}{2} \Delta_{R}\left(k^{0}\right) \Delta_{A}\left(k^{0}\right)=-\frac{\rho\left(k^{0}\right)}{2 \operatorname{Im} \Sigma\left(k^{0}, \mathbf{k}\right)},
$$

which is proportional to $N$ in the large $N$ limit. Note that the other contributions [as well as the whole product $\rho\left(k^{0}\right) \rho\left(k^{0}-\omega\right)$ for $\beta \omega \gg(1 / N)$ ] give contributions of order $\mathcal{O}\left(N^{0}\right)$, bringing us back to the $R 0$ result (19). The noncommutative nature of the limits $\beta \omega \rightarrow 0$, $(1 / N) \rightarrow 0$ imply that for the calculation of transport coefficients, contributions that are naively subleading at large $N$ become important. This enhancement process, known as "pinching poles," has a long history in nuclear physics literature, cf. Refs. [19,28].

In the small frequency limit, the $R 2$-level expression for the shear viscosity from Eq. (3) is

$$
\eta_{R 2}=\left.\frac{N}{16 \pi} \int_{m}^{\infty} d E \frac{k^{4} n^{\prime}(E)}{\operatorname{Im} \Sigma(E)}\right|_{k=\sqrt{E^{2}-m^{2}}} .
$$

The $R 2$-level expression for the shear viscosity is well defined for all values of the coupling, but it does not contain all contributions to leading order in large $N$. To this end, let us reconsider the correlator $G_{E}^{x y, x y}$ in $R$ 4-level resummation, specified by (11) with (17). Since the $R 4$-level action contains a nontrivial vertex, Wick's theorem can no longer be used to evaluate the correlator; instead, interactions must be included (see the Supplemental Material for an example of how a generic four-point function is evaluated beyond $R 2$ [22]). For the energy-momentum correlator $G_{E, R 4}^{x y, x y}$, the situation is slightly more simple than for a generic fourpoint function, because the spatial derivatives $\partial_{x} \phi, \partial_{y} \phi$ in the definition imply that some contributions do not contribute after angular integration (see Supplemental Material [22]). However, instead of the regular three-vertex $\Gamma_{3}$, the corresponding contribution to $G_{E, R 4}^{x y, x y}$ contains momenta $k_{x}$, $k_{y}$ inside the vertex. One thus finds

$G_{E, R 4}^{x y, x y}\left(p_{E}\right)=2 N \bigvee_{K} k_{x} k_{y} \Gamma_{3, x y}(K, P-K) \Delta(K) \Delta(P-K)$, where $\Gamma_{3, x y}=k_{x} k_{y}+\delta \Gamma_{3, x y}$ denotes the resummed threevertex, and the propagator $\Delta(K)$ contains $\Sigma$ in the $R 4$-level resummation, cf. Eq. (15). The resummed vertex is the only modification with respect to the $R 2$ result (20), so one needs to check if $\delta \Gamma_{3, x y}$, which naively is order $1 / N$, gets enhanced in the limit $P \rightarrow 0$. One finds

$$
\begin{aligned}
\delta \Gamma_{3, x y}(K, P-K)= & -4 \bigvee_{Q} \Delta(Q) \Delta(P-Q) W(P, Q, K) \\
& \times \Gamma_{3}(-Q, P-K) \Gamma_{3}(K, Q-P) \\
& \times \Gamma_{3, x y}(Q, P-Q),
\end{aligned}
$$

where the integral kernel is $W(P, Q, K)=$ $D(P-K-Q)+2 N \Gamma_{4}(Q, P-Q, K, P-K)$. The structure of (27) indeed suggests a pinching pole similar to (20) in the limit $P \rightarrow 0$, whereas for other kinematic regions $\delta \Gamma_{3, x y} \propto(1 / N)$. Two of the internal vertices in (27) therefore do not receive corrections in the limit $P \rightarrow 0$. One may verify that, in this limit, $\delta \Gamma_{3, x y}(K,-K)=\left(k_{x} k_{y} / k^{2}\right) \bar{\Gamma}_{3}(K)$, such that after doing the angular average one obtains

$\left.G_{E, R 4}^{x y, x y}\left(p_{E}\right)=\frac{N}{4}\right\}_{K} k^{2} \bar{\Gamma}_{3}(K, P-K) \Delta(K) \Delta(P-K)$,

where to leading order in large $N$

$$
\begin{aligned}
\bar{\Gamma}_{3}(K, P-K)= & k^{2}-4 \chi_{Q} \Delta(Q) \Delta(P-Q) W(P, Q, K) \\
& \times \bar{\Gamma}_{3}(Q, P-Q) \times\left[2(\hat{\mathbf{q}} \cdot \hat{\mathbf{k}})^{2}-1\right] .
\end{aligned}
$$

The analytic structure of the vertex $\bar{\Gamma}_{3}(K, P-K)$ is as follows: Expressing the propagators in terms of their spectral functions $\rho(\mu), \rho_{D}(\mu)$, one can perform the analytic continuation to real frequencies. In a first step, setting $\bar{\Gamma}_{3}(Q, P-Q)=q^{2}$, the thermal sum over $q_{E}$ in (27) can be done explicitly. Since the integrations over the arguments of the spectral functions range over the whole real axis, one finds that $\bar{\Gamma}_{3}\left(i k^{0}, i p^{0}-i k^{0}\right)$ has branch cuts along the whole real line for $\operatorname{Im} p^{0}=0, \operatorname{Im} k^{0}=0$, and $\operatorname{Im}\left(p^{0}-k^{0}\right)=0$. This structure is unchanged when iterating the vertex.

With the analytic structure of the vertex known, one proceeds to evaluate (28). First, write the thermal sum as

$$
T \sum_{k_{E}} f\left(k_{E}\right)=\oint_{\mathcal{C}} \frac{d k^{0}}{4 \pi i} \operatorname{coth} \frac{\beta k^{0}}{2} f\left(i k^{0}\right),
$$

with $\mathcal{C}$ encircling the poles at the imaginary Matsubara frequencies $k^{0}=i k_{E}$ in a counterclockwise fashion. Next, the propagators $\Delta(K) \Delta(P-K)$ have branch cuts at $\operatorname{Im} k^{0}=0$ and $\operatorname{Im}\left(k^{0}\right)=-p_{E}$. The additional branch cut from the vertex is independent from $k^{0}$ and hence not relevant for the thermal sum (30). Deforming the contour $\mathcal{C}$ 
such that it runs along both sides of the two branch cuts, one encounters terms such as

$$
\bar{\Gamma}_{3}\left(i k^{0} \pm 0^{+}, p_{E}-i k^{0}\right) \Delta\left(i k^{0} \pm 0^{+}\right) \Delta\left(p_{E}-i k^{0}\right) .
$$

Upon analytic continuation $p_{E} \rightarrow i p^{0}-0^{+}$, one recognizes the retarded and advanced correlators $\Delta_{R, A}$ using (1), where $\Delta_{R}\left(-k^{0}\right)=\Delta_{A}\left(k^{0}\right)$. The leading large $N$ contributions are then given by combinations such as $\Delta_{R}\left(k^{0}\right) \Delta_{A}\left(k^{0}-\omega\right)$, which have singularities on either side of the real $k^{0}$ axis (pinching poles), whereas the others can be neglected. Letting $k^{0} \rightarrow k^{0}-p^{0}$ in the second branch cut contribution, only a particular analytic continuation of the vertex function contributes, which for $p^{0} \rightarrow 0$ and $k^{0}= \pm E_{k}$ becomes

$\left.\lim _{p^{0} \rightarrow 0} \bar{\Gamma}_{3}\left(i k^{0}-0^{+}, i\left(p^{0}-k^{0}\right)-0^{+}, \mathbf{k}\right)\right|_{k^{0}= \pm E_{k}} \equiv F\left(E_{k}\right)$.

Clearly, this corresponds to using standard analytic continuation of $\bar{\Gamma}_{3}(K, P-K)$ first for $k_{E}$ and then for $p_{E}$. Assuming that $F\left(E_{k}\right)$ is real (which will be shown below), one can then follow the same procedure that led to (25), with the only modification arising from the resummed vertex function. Using (10), one finds

$$
\underline{\eta}=\frac{1}{4} \frac{\int_{m}^{\infty} d E \frac{F(E)}{\beta \operatorname{Im} \Sigma(E)} k^{2} n^{\prime}(E)}{-\int_{m}^{\infty} d E E k^{2} n^{\prime}(E)},
$$

where again $k=\sqrt{E^{2}-m^{2}}$. This relation is exact in the large $N$ limit, as it contains all leading order large $N$ contributions to the shear viscosity and entropy density, cf. Ref [19]. Note that, because $\Sigma \propto(1 / N)$, one finds that $(\eta / s) \propto N$ in the large $N$ limit. This $N$ scaling is generic for vector or fermionic theories [12,19], but is qualitatively different from large $N$ gauge theories where $\eta / s \propto$ $\mathcal{O}\left(N^{0}\right)[6,9]$.

In order to get a result for $\eta / s$, one needs to know the functions $\operatorname{Im} \Sigma(E)$ and $F(E)$. These follow from finitetemperature field theory calculations, see Supplemental Material [22] and Refs. [19,29-31]. For the evaluation of $\eta / s$, it is convenient to use quadrature to recast the integrals in terms of sums (see Supplemental Material [22]). To this end, construct orthogonal polynomials $P_{n}(x)$ of degree $n$

$$
-\int_{m}^{\infty} d E n^{\prime}(E) P_{n}(E) P_{m}(E)=\delta_{n, m},
$$

for $\quad n=0,1, \ldots, K$. Expanding $\quad\left[F\left(E_{k}\right) / \operatorname{Im} \Sigma\left(E_{k}\right)\right]=$ $\sum_{n=0}^{\infty} b_{n} P_{n}\left(E_{k}\right)$ only the coefficients $b_{0}, b_{1}$, and $b_{2}$ contribute to $\eta / s$ because $k^{2}$ in the numerator of (33) only involves polynomials $P_{n}(E)$ up to degree 2 .

Results and universality at infinite coupling.Numerical evaluation of (33) for all values of the dimensionless coupling $\hat{\lambda}=\beta \lambda$ is shown in Fig. 1. For weak

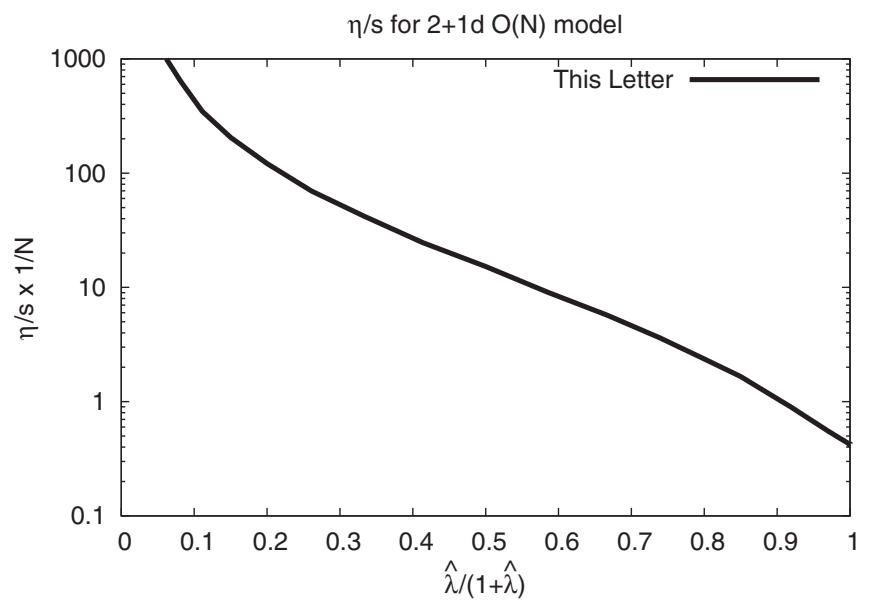

FIG. 1. Shear viscosity over entropy density as a function of the dimensionless coupling $\hat{\lambda} \equiv \beta \lambda$ in the $2+1 d O(N)$ model with quartic self-interaction. Horizontal axis is compactified in order to fit values $\hat{\lambda} \in[0, \infty)$. The numerical code to calculate this result is publicly available from Ref. [32].

couplings $\hat{\lambda}, \eta / s N$ is large because the thermal width is small. As the coupling is increased, I find that the ratio of shear viscosity over entropy density is dropping monotonically, but is finite in the limit of $\hat{\lambda} \rightarrow \infty$. In this strongcoupling limit, the numerically calculated result becomes

$$
\lim _{\hat{\lambda} \rightarrow \infty} \frac{\eta}{S}=0.42(1) \times N
$$

One may ask about the universality of the strongcoupling result. To this end, consider a modification of the action (4) to

$$
S=\int d^{d} X\left(\frac{1}{2} \partial_{\mu} \phi_{a} \partial_{\mu} \phi_{a}+\frac{\lambda}{N^{2}}\left(\phi_{a} \phi_{a}\right)^{3}\right)
$$

where now $\lambda$ is dimensionless. This action has the property that it is a conformal field theory for all values of $\lambda$ at large $N$ [16]. Using the same replacement $\sigma=\phi_{a} \phi_{a}$ as before and integrating out $\sigma$, one finds

$$
Z=\int \mathcal{D} \phi \mathcal{D} \zeta e^{-\int_{X}\left(\frac{1}{2} \partial_{\mu} \phi_{a} \partial_{\mu} \phi_{a}+i \zeta \phi_{a} \phi_{a}-\ln \left\{\operatorname{Ai}\left[i \zeta\left(\frac{N^{2}}{3 \lambda}\right)^{\frac{1}{3}}\right]\right\}\right)} .
$$

At large $N$, the asymptotic properties of the Airy function then give the form of the partition function as

$$
Z=\int \mathcal{D} \phi \mathcal{D} \zeta e^{-\int_{X}\left[\frac{1}{2} \partial_{\mu} \phi_{a} \partial_{\mu} \phi_{a}+i \zeta \phi_{a} \phi_{a}+\frac{2}{3 \sqrt{3 \pi}} N(i \zeta)^{\left.\frac{3}{2}\right]}\right.} .
$$

While it may be challenging to construct $R 4$ for this Lagrangian for general values of $\lambda$, the strong-coupling limit $\lambda \rightarrow \infty$ of this theory is exactly equal to the strongcoupling limit $\hat{\lambda} \rightarrow \infty$ of Eq. (5). For this reason, one can 
explicitly write down $R 4$ for this theory in the strongcoupling limit, where the only difference is the form of the propagator

$$
D(K)=\frac{1}{N \Pi(K)},
$$

cf. Eq. (11). Hence in the infinite coupling limit, the result for the shear viscosity for (36) is identical to that of (4). It is not hard to generalize this proof to theories with other interactions $\phi_{a} \phi_{a} \rightarrow U\left(\phi_{a} \phi_{a}\right)$ for a large class of potentials $U(x)$, which demonstrates that (35) is the universal strongcoupling shear viscosity over entropy ratio for a large class of bosonic quantum field theories. The same universal behavior was found [16] for the weak-strong ratio $\left[\left(s_{\lambda=\infty}\right) /\left(s_{\lambda=0}\right)\right]=\frac{4}{5}$ and for the boson in-medium mass $\lim _{\lambda \rightarrow \infty} \hat{m}=2 \ln [(1+\sqrt{5}) / 2]$.

Summary and discussion.- In this Letter, I derived an exact large $N$ expression for the ratio of shear viscosity over entropy density for the interacting $O(N)$ model, using wellestablished field theory techniques. Evaluating the expression numerically in the case of $2+1$ dimensions, I found the strong-coupling result (35).

Regardless of the numerical value, the present Letter demonstrates that it is possible to calculate transport properties at infinite coupling directly from quantum field theory, without invoking dualities or conjectures of any kind. While the field theory studied here may not be of interest to most readers, it can nevertheless serve as a test bed for strong-coupling transport, which would otherwise be inaccessible or very hard by any other means. For instance, having access to exact full energy-momentum tensor correlation functions for all values of the coupling allows one to study the onset and breakdown of hydrodynamics from first principles, cf. Refs. [33-35]; exact real-time correlators also can be used to test analytic continuation techniques employed in lattice Monte Carlo studies $[13,14]$, and exact results for transport coefficients can be used as a rigorous test case for approximation schemes that are used for, e.g., QCD [9-11].

In addition to serving as a well-defined test bed for general-purpose tools, the present calculation may be generalized in several ways: by, for instance, calculating other transport coefficients such as the bulk viscosity $\zeta$ as well as relaxation time $\tau_{\pi}$ for the $O(N)$ model, calculating transport coefficients for other large $N$ theories [36-38], and calculating exact far-from-equilibrium real-time dynamics at large $N$ for a quantum field theory.

For these reasons, I am optimistic that the present result can become useful in the future.

I am indebted to Gert Aarts for clarifying some questions I had concerning Ref. [19], as well as providing numerical data for $\eta / s$ in the 4D $O(N)$ model from this reference.
Also, I thank Scott Lawrence and Max Weiner for fruitful discussions and Marcus Pinto for pointing out a typo in (38). This work was supported by the Department of Energy, DOE Award No. DE-SC0017905.

[1] P. Romatschke and U. Romatschke, Viscosity Information from Relativistic Nuclear Collisions: How Perfect is the Fluid Observed at RHIC? Phys. Rev. Lett. 99, 172301 (2007).

[2] K. Dusling and D. Teaney, Simulating elliptic flow with viscous hydrodynamics, Phys. Rev. C 77, 034905 (2008).

[3] B. Schenke, S. Jeon, and C. Gale, Elliptic and Triangular Flow in Event-by-Event $(3+1) D$ Viscous Hydrodynamics, Phys. Rev. Lett. 106, 042301 (2011).

[4] H. Song, S. Bass, and U. W. Heinz, Spectra and elliptic flow for identified hadrons in $2.76 \mathrm{~A} \mathrm{TeV} \mathrm{Pb}+\mathrm{Pb}$ collisions, Phys. Rev. C 89, 034919 (2014).

[5] J. E. Bernhard, J. S. Moreland, and S. A. Bass, Bayesian estimation of the specific shear and bulk viscosity of quarkgluon plasma, Nat. Phys. 15, 1113 (2019).

[6] G. Policastro, D. T. Son, and A. O. Starinets, The Shear Viscosity of Strongly Coupled $N=4$ Supersymmetric Yang-Mills Plasma, Phys. Rev. Lett. 87, 081601 (2001).

[7] Y. Kats and P. Petrov, Effect of curvature squared corrections in AdS on the viscosity of the dual gauge theory, J. High Energy Phys. 01 (2009) 044.

[8] A. Buchel, Resolving disagreement for $\eta / s$ in a CFT plasma at finite coupling, Nucl. Phys. B803, 166 (2008).

[9] P. B. Arnold, G. D. Moore, and L. G. Yaffe, Transport coefficients in high temperature gauge theories. 1. Leading log results, J. High Energy Phys. 11 (2000) 001.

[10] P. B. Arnold, G. D. Moore, and L. G. Yaffe, Transport coefficients in high temperature gauge theories. 2. Beyond leading log, J. High Energy Phys. 05 (2003) 051.

[11] J. Ghiglieri, G. D. Moore, and D. Teaney, QCD shear viscosity at (almost) NLO, J. High Energy Phys. 03 (2018) 179.

[12] G. D. Moore, Transport coefficients in large $N(f)$ gauge theory: Testing hard thermal loops, J. High Energy Phys. 05 (2001) 039.

[13] H. B. Meyer, A Calculation of the shear viscosity in SU(3) gluodynamics, Phys. Rev. D 76, 101701(R) (2007).

[14] S. Borsányi, Z. Fodor, M. Giordano, S. D. Katz, A. Pásztor, C. Ratti, A. Schäfer, K. K. Szabó, and B. C. Tóth, High statistics lattice study of stress tensor correlators in pure SU(3) gauge theory, Phys. Rev. D 98, 014512 (2018).

[15] P. Romatschke, Analytic transport from weak to strong coupling in the $O(N)$ model, Phys. Rev. D 100, 054029 (2019).

[16] P. Romatschke, Finite-Temperature Conformal Field Theory Results for All Couplings: $O(N)$ Model in 2+1 Dimensions, Phys. Rev. Lett. 122, 231603 (2019); Erratum, Phys. Rev. Lett. 123, 209901 (2019).

[17] P. Romatschke, Simple non-perturbative resummation schemes beyond mean-field: Case study for scalar $\phi^{4}$ theory in $1+1$ dimensions, J. High Energy Phys. 03 (2019) 149.

[18] P. Romatschke, Simple non-perturbative resummation schemes beyond mean-field II: Thermodynamics of scalar 
$\phi^{4}$ theory in $1+1$ dimensions at arbitrary coupling, Mod. Phys. Lett. A 35, 2050054 (2020).

[19] G. Aarts and J. M. Martinez Resco, Shear viscosity in the $O(N)$ model, J. High Energy Phys. 02 (2004) 061.

[20] D. Forster, D. R. Nelson, and M. J. Stephen, Long-Time Tails and the Large-Eddy Behavior of a Randomly Stirred Fluid, Phys. Rev. Lett. 36, 867 (1976).

[21] P. Kovtun, G. D. Moore, and P. Romatschke, The stickiness of sound: An absolute lower limit on viscosity and the breakdown of second order relativistic hydrodynamics, Phys. Rev. D 84, 025006 (2011).

[22] See Supplemental Material at http://link.aps.org/ supplemental/10.1103/PhysRevLett.127.111603 for technical details of the calculation.

[23] P. Kovtun, Lectures on hydrodynamic fluctuations in relativistic theories, J. Phys. A 45, 473001 (2012).

[24] P. Romatschke and U. Romatschke, Relativistic Fluid Dynamics In and Out of Equilibrium, Cambridge Monographs on Mathematical Physics (Cambridge University Press, Cambridge, England, 2019).

[25] W. Florkowski, M. P. Heller, and M. Spalinski, New theories of relativistic hydrodynamics in the LHC era, Rep. Prog. Phys. 81, 046001 (2018).

[26] M. Laine and A. Vuorinen, Basics of Thermal Field Theory (Springer, New York, 2016), Vol. 925.

[27] S. Sachdev, Polylogarithm identities in a conformal field theory in three-dimensions, Phys. Lett. B 309, 285 (1993).

[28] S. Jeon, Hydrodynamic transport coefficients in relativistic scalar field theory, Phys. Rev. D 52, 3591 (1995).
[29] M. A. Valle Basagoiti, Transport coefficients and ladder summation in hot gauge theories, Phys. Rev. D 66, 045005 (2002).

[30] I. R. Klebanov and A. M. Polyakov, AdS dual of the critical $O(N)$ vector model, Phys. Lett. B 550, 213 (2002).

[31] P. Romatschke and M. Strickland, Collective modes of an anisotropic quark gluon plasma, Phys. Rev. D 68, 036004 (2003).

[32] P. Romatschke, $O(N)$ shear viscosity calculator, https:// github.com/paro8929/etaON.

[33] P. Romatschke, Retarded correlators in kinetic theory: Branch cuts, poles and hydrodynamic onset transitions, Eur. Phys. J. C 76, 352 (2016).

[34] S. Grozdanov, N. Kaplis, and A. O. Starinets, From strong to weak coupling in holographic models of thermalization, J. High Energy Phys. 07 (2016) 151.

[35] M. P. Heller, A. Serantes, M. Spaliński, V. Svensson, and B. Withers, Transseries for causal diffusive systems, J. High Energy Phys. 04 (2021) 192.

[36] O. DeWolfe and P. Romatschke, Strong coupling universality at large $N$ for pure CFT thermodynamics in $2+1$ dimensions, J. High Energy Phys. 10 (2019) 272.

[37] M. B. Pinto, Three dimensional Yukawa models and CFTs at strong and weak couplings, Phys. Rev. D 102, 065005 (2020).

[38] P. Romatschke and M. Säppi, Thermal free energy of large $N_{f}$ QED in $2+1$ dimensions from weak to strong coupling, Phys. Rev. D 100, 073009 (2019). 